\title{
Avaliação Ultra-sonográfica e Urodinâmica em Pacientes com Incontinência Urinária
}

\author{
Ultrasonographic and Urodynamic Evaluation of Patients with Urinary Incontinence
}

\author{
Marair Gracio Ferreira Sartori, Carlos Alberto Salles Bergamo \\ Sérgio Brasileiro Martins, Cláudia Takano \\ Manoel João Batista Castello Girão \\ Edmund Chada Baracat, Geraldo Rodrigues de Lima
}

\begin{abstract}
RESUMO
Objetivos: avaliar a concordância entre os diagnósticos urodinâmico e ultra-sonográfico de incontinência urinária, bem como correlacionar as variáveis de ambos os exames.

Metodologia: Foram selecionadas 381 pacientes com perda de urina, entre as atendidas no Setor de Uroginecologia e Cirurgia Vaginal da Disciplina de Ginecologia da Escola Paulista de Medicina - Universidade Federal de São Paulo. Todas foram submetidas a estudo urodinâmico, seguindo a padronização da Sociedade Internacional de Continência, e ultrasonografia do colo vesical, com transdutor endovaginal de $6 \mathrm{MHz}$. No estudo urodinâmico, medimos a pressão máxima de fechamento uretral (PMFU) e obtivemos o diagnóstico etiológico da perda de urina. As mulheres foram agrupadas segundo o diagnóstico urodinâmico em incontinência urinária de esforço, instabilidade do detrusor e incontinência urinária mista. Na ultra-sonografia, foram avaliados a posição do colo vesical em relação à borda inferior da sinfise púbica e sua mobilidade e os diâmetros da uretra e do colo vesical.

Resultados: 1) o colo vesical, quando em repouso, esteve mais freqüentemente acima da borda inferior da sinfise púbica e, durante o esforço, abaixo ou na sua altura nos três grupos; 2) a mobilidade do colo vesical foi semelhante nos grupos; 3) não houve correlação significante entre PMFU e diâmetro da uretra e do colo vesical, tanto em repouso quanto ao esforço.

Conclusão: a ultra-sonografia do colo vesical é sempre complementar à avaliação clínica e ao estudo urodinâmico.
\end{abstract}

PALAVRAS-CHAVE: Incontinência urinária. Estudo urodinâmico. Ultra-sonografia transvaginal. Uretra.

Trabalho realizado no Setor de Uroginecologia e Cirurgia Vaginal da Disciplina de Ginecologia da Escola Paulista de Medicina Universidade Federal de São Paulo

Correspondência:

Marair G. F. Sartori

Av. Onze de Junho, 1006 apto 51 - Vila Mariana

04041-003 - São Paulo - SP

\section{Introdução}

A incontinência urinária, definida como toda perda de urina que ocasione problema social ou higiênico para a mulher ${ }^{3}$, é uma das afecções mais 
freqüentes nos serviços de ginecologia ${ }^{8}$.

A incontinência urinária de esforço na mulher decorre de vários fatores, porém, associa-se com maior constância à descida rotacional da uretra ou à posição extrabdominal do colo vesical. Desta forma, aumentos da pressão abdominal não se transmitem à uretra, resultando em perda de urina ${ }^{3}$.

O estudo urodinâmico (EUD) é um dos principais recursos subsidiários na avaliação da incontinência urinária; detecta contrações não-inibidas, que caracterizam instabilidade do detrusor, e que é passivel de tratamento clínico. A perda de urina sincrônica ao esforço, na ausência de atividade do detrusor detectada pelo EUD, caracteriza a incontinênca urinária de esforço. Contudo, o exame urodinâmico, isoladamente, não avalia a mobilidade do colo e a situação da junção uretrovesical, quer em repouso, quer aos esforços. Para tanto, é necessário que se associem métodos de imagem ao EUD, tais como a uretrocistografia miccional ou a ultra-sonografia do colo vesical ${ }^{7}$.

A uretrocistografia miccional com correntinha foi um dos primeiros métodos utilizados no diagnóstico da incontinência urinária; analisa a posição do colo vesical durante o repouso e ao esforço, bem como determina os ângulos de inclinação uretral e uretrovesical posterior ${ }^{2}$. Nos dias atuais, leva-se em consideração para o diagnóstico apenas a situação e a posição do colo vesical, já que há ampla superposição entre os valores destes ângulos medidos em indivíduos normais e em incontinentes ${ }^{7}$. O exame, também, apresenta grandes desvantagens, como a dificuldade técnica, o desconforto para a paciente e a exposição à radiação ionizante. Por isso, salvo situações especiais, não deve mais ser solicitado.

Têm sido descritas várias técnicas de exame ultra-sonográfico com a finalidade de avaliar a mobilidade do colo. Algumas utilizam transdutores por via retal, outras por via vaginal ou perineal, quer em posição ortostática ou ginecológica.

Kohorn et al. ${ }^{5}$ estudaram a mobilidade do colo vesical de 30 mulheres incontinentes, por meio da ultra-sonografia e da uretrocistografia miccional. Obtiveram resultados semelhantes com ambos os métodos. Nesse estudo, utilizaram transdutor linear de 3,5 MHz em posição sagital, na vulva, o que permitiu adequada visibilização da sinfise púbica, da uretra e da base vesical.

Ribeiro ${ }^{7}$ estudou, mediante de ultrasonografia vaginal, 35 mulheres com incontinência urinária de esforço, comparando-as a 22 continentes. Para tanto, realizou o exame ultrasonográfico com a paciente em posição ortostática e ligeira abdução da articulação coxo-femoral, em repouso e durante esforço. Utilizou transdutor transvaginal, introduzido pela própria mulher, até cerca de $2 \mathrm{~cm}$ do intróito. Mediu a distância do colo vesical até a borda inferior da sínfise púbica. Observou que a mobilidade do colo vesical foi maior nas pacientes incontinentes, demonstrando a importância do método no diagnóstico.

No Setor de Uroginecologia e Cirurgia Vaginal da Disciplina de Ginecologia da Escola Paulista de Medicina temos realizado a ultra-sonografia como método complementar no diagnóstico dos distúrbios urinários.

Propusemo-nos, no presente estudo, a avaliar os diagnósticos urodinâmico e ultra-sonográfico de incontinência urinária e a correlação entre as variáveis de ambos os métodos.

\section{Pacientes e Métodos}

Foram incluídas neste estudo 381 pacientes com queixa de perda de urina atendidas no Setor de Uroginecologia e Cirurgia Vaginal da Disciplina de Ginecologia da Escola Paulista de Medicina Universidade Federal de São Paulo, no período de 1995 a 1996.

Todas as mulheres submeteram-se, dentro da rotina de atendimento no setor, a pormenorizada anamnese, exames físico geral e ginecológico, bem como ao estudo urodinâmico e à ultra-sonografia do colo vesical.

O estudo urodinâmico foi realizado com aparelho de quatro canais, com água destilada a velocidade média de infusão e temperatura ambiente, segundo padronização da Sociedade Internacional de Continência ${ }^{3}$.

As mulheres foram agrupadas, segundo o exame, em: 1) incontinência urinária de esforço (IUE), quando se notou perda de urina simultânea ao esforço solicitado, durante a fase de enchimento vesical, sem atividade do detrusor (351 casos); 2) instabilidade do detrusor (INST), quando se registraram contrações do músculo detrusor com mais de $15 \mathrm{~cm} \mathrm{H}_{2} \mathrm{O}$ ou de menor amplitude, porém acompanhada de perda de urina (13 casos) e 3) incontinência urinária mista (IUM), quando houve associação de ambas as situações (17 casos).

A ultra-sonografia do colo vesical foi realizada com transdutor endovaginal de $6 \mathrm{MHz}$, envolto por preservativo lubrificado com gel sônico, colocado no intróito vaginal, logo abaixo do clitóris, estando a paciente em posição ginecológica. Esta posição favorece a segura identificação da sínfise púbica, da uretra, do colo, da base vesical e da bexiga.

Foi medida a distância entre a junção uretrovesical e a borda inferior da sínfise púbica 
em repouso. A seguir, pedia-se que a paciente realizasse manobra de Valsalva, medindo-se a nova distância entre essas estruturas. Dessa maneira, pode-se verificar a posição do colo vesical em repouso e durante o esforço, em relação à borda inferior da sinfise púbica, bem como a sua mobilidade. Mediu-se o diâmetro do colo vesical e da uretra a $1 \mathrm{~cm}$ do colo vesical, em repouso e durante o esforço.

Para a análise dos resultados foi aplicado: o teste de McNemar, com a finalidade de comparar os resultados nas situações de repouso e de esforço entre as pacientes com os diferentes diagnósticos. Também foram empregados o teste do $\chi^{2} \mathrm{e}$ coeficiente de concordância kappa.

Em todos os testes fixou-se em 0,05 ou $5 \%$ o nível de rejeição da hipótese de nulidade.

\section{Resultados}

Observa-se, na Tabela 1, que entre as pacientes com diagnóstico urodinâmico de IUE, $62,1 \%$ apresentaram colo vesical abaixo ou na borda inferior da sínfise púbica durante o esforço, ao passo que, em $37,9 \%$, o colo achava-se acima desta. Realizou-se análise estatística pelo teste de McNemar, avaliando-se os casos com colo vesical acima e abaixo da borda inferior da sínfise púbica, em repouso e durante o esforço, para os grupos de pacientes com IUE, INST e IUM (Tabelas 1, 2 e 3). Demonstrou-se que, nos três grupos, é significantemente mais freqüente o colo vesical estar, em repouso, acima da borda inferior da sínfise púbica e, durante o esforço, estar abaixo ou na altura da referência óssea.

Analisando-se a Tabela 4, observa-se que $61,5 \%$ dos casos com instabilidade do detrusor apresentam mobilidade do colo vesical maior do que $10 \mathrm{~mm}$. No grupo com IUE, somente 30,5\% não apresentaram deslocamento significativo do colo vesical, porém, a maior parte dos casos analisados $(69,5 \%)$ apresentou hipermobilidade do colo, independentemente do estudo urodinâmico. A análise pelo teste do $\chi^{2}$ não evidenciou diferenças entre os grupos.

Finalmente, relacionou-se a pressão máxima de fechamento uretral com o diâmetro da uretra e do colo vesical, em repouso e durante o esforço (Tabela 5), não se detectando correlação significante.
Tabela 1 - Posição do colo vesical em relação à borda inferior da sínfise púbica, avaliada pela ultra-sonografia nas pacientes com incontinência urinária de esforço, em repouso e durante o esforço

\begin{tabular}{crccr} 
Em repouso & \multicolumn{3}{c}{ Durante esforço } & Total \\
\cline { 2 - 4 } & Acima & Abaixo & No nível & \\
\hline Acima & 133 & 137 & 63 & 333 \\
Abaixo & 0 & 10 & 0 & 10 \\
Nível & 0 & 0 & 0 & 8 \\
Total & 133 & 8 & 63 & 351 \\
\hline
\end{tabular}

IUE= Incontinência urinária de esforço

Coeficiente de concordância KW Teste de McNemar

Tabela 2 - Posição do colo vesical em relação à borda inferior da sínfise púbica, avaliada pela ultra-sonografia, nas pacientes com instabilidade vesical, em repouso e durante esforço

\begin{tabular}{ccccc} 
Em repouso & \multicolumn{3}{c}{ Durante esforço } & Total \\
\cline { 2 - 4 } & Acima & Abaixo & No nível & \\
\hline Acima & 6 & 5 & 1 & 12 \\
Abaixo & 0 & 1 & 0 & 1 \\
Nível & 0 & 0 & 0 & 0 \\
Total & 6 & 6 & 1 & 13 \\
\hline & Coeficiente de concordância KW \\
Teste de McNemar
\end{tabular}

Tabela 3 - Posição do colo vesical em relação à borda inferior da sínfise púbica, avaliada pela ultra-sonografia, nas pacientes com incontinência urinária mista, em repouso e durante esforço

\begin{tabular}{ccccc} 
& \multicolumn{3}{c}{ Durante esforço } & Total \\
\cline { 2 - 4 } & Acima & Abaixo & No nível & \\
\hline Acima & 6 & 9 & 2 & 17 \\
Abaixo & 0 & 0 & 0 & 0 \\
Nível & 0 & 0 & 0 & 0 \\
Total & 6 & 9 & 2 & 17
\end{tabular}

Coeficiente de concordância KW: Zcalc $=1,08$

Teste de McNemar: $\chi_{\text {calc }}^{2}=6,00^{*}(p<0,02)$

Tabela 4 - Mobilidade do colo vesical detectada pela ultra-sonografia, segundo o diagnóstico urodinâmico

\begin{tabular}{crrrr}
\hline \multirow{2}{*}{ Diagnóstico } & \multicolumn{3}{c}{ Mobilidade } & Total \\
\cline { 2 - 4 } & até $10 \mathrm{~mm}$ & $>10 \mathrm{~mm}$ & $\begin{array}{c}\% \text { de até } \\
10 \mathrm{~mm}\end{array}$ \\
\hline INST & 5 & 8 & 38,5 & 13 \\
IUE & 107 & 244 & 30,5 & 351 \\
IUM & 6 & 11 & 35,5 & 17 \\
Total & 118 & 263 & 31,0 & 381 \\
\hline
\end{tabular}

Inst = instabilidade do detrusor

IUE= incontinência urinária de esforço IUM = incontinência urinária mista 
Tabela 5 - Correlação entre a pressão máxima de fechamento uretral (PMFU), em centímetros de água, e os diâmetros da uretra e do colo vesical em milímetros, dos casos estudados, em repouso e durante esforço.

\begin{tabular}{ccccc}
\hline & $\begin{array}{c}\text { colo } \\
\text { repouso }\end{array}$ & $\begin{array}{c}\text { colo } \\
\text { esforço }\end{array}$ & $\begin{array}{c}\text { uretra } \\
\text { repouso }\end{array}$ & $\begin{array}{c}\text { uretra } \\
\text { esforço }\end{array}$ \\
\hline PMFU & Média & Média & No nível & No nível \\
\hline$\leq 25$ & 4.6 & 4,1 & 4.6 & 4.7 \\
$>25 \leq 40$ & 5.5 & 5.2 & 5.3 & 4.6 \\
$>25 \leq 80$ & 5.2 & 5.1 & 5.2 & 5.3 \\
$>60 \leq 80$ & 5.1 & 5.1 & 5.3 & 5.0 \\
$>80$ & 5.7 & 5,7 & 5.7 & 5.5 \\
\hline
\end{tabular}

Coeficiente de correlação de Spearman: não significante

\section{Discussão}

A importância do estudo urodinâmico na avaliação da perda de urina é inegável ${ }^{3}$, não apenas pela possibilidade de erro quando se considera apenas o diagnóstico clínico ${ }^{6}$, mas também por permitir identificar as mulheres com defeito esfincteriano uretral, cujo tratamento requer técnicas cirúrgicas específicas ${ }^{3}$.

A ultra-sonografia do colo vesical tem sido bastante utilizada na avaliação da incontinência urinária, identificando basicamente as mulheres que apresentam hipermobilidade do colo vesical, ou seja, deslocamentos maiores do que $10 \mathrm{~mm}^{7}$. Entretanto, o uso da ultra-sonografia como método isolado para o diagnóstico da incontinência urinária é bastante discutido ${ }^{1,9}$.

Pelos nossos resultados, verifica-se que a grande maioria das mulheres incontinentes apresentou hipermobilidade do colo vesical, independentemente do tipo de perda urinária, ou seja, o fato de o diagnóstico final ser de instabilidade vesical, incontinência urinária de esforço ou incontinência urinária mista não está relacionado com a posição do colo vesical. Isso faz supor que, se levássemos em consideração apenas a mobilidade do colo vesical, estariamos indicando tratamento cirúrgico em mulheres que, na verdade, apresentam instabilidade do detrusor ${ }^{4,7}$.

Ao empregarmos o critério da posição do colo vesical, em repouso e durante o esforço, em relação à borda inferior da sinfise púbica, observamos que as mulheres com instabilidade do detrusor podem apresentar o colo infrapúbico durante o esforço. No entanto, as pacientes com incontinência urinária de esforço apresentam com maior freqüência o colo nessa situação.

O estudo urodinâmico, por meio do perfil pressórico uretral, nos dá informações de grande importância em relação à integridade do sistema esfincteriano da uretra. Considera-se sugestivo de defeito esfincteriano o encontro de pressão máxima de fechamento menor ou igual a $25 \mathrm{cmH}_{2} \mathrm{O}^{3}$. No presente estudo, procuramos verificar se a pressão máxima de fechamento uretral se correlacionava com o diâmetro da uretra ou do colo vesical, mas não foi possivel detectar tal correlação.

Julgamos, assim, pelos nossos resultados, que a ultra-sonografia do colo vesical é sempre complementar à avaliação clínica e ao estudo urodinâmico.

\section{SUMMARY}

Purpose: to evaluate the agreement between the urodynamic and ultrasonography diagnoses of urinary incontinence, as well as to correlate the variables of both examinations.

Methodology: three hundred eighty-one patients with urine loss were selected, from the Sectior of Urogynecology and Vaginal Surgery of the Division of Gynecology, Escola Paulista de Medicina - Federal University of São Paulo. All of them were submitted to urodynamic study, according to the standardization of the International Society of Continence, and to ultrasonography of the bladder neck, with a $6 \mathrm{MHz}$ trasvaginal transducer. We analyzed the maximum closing urethral pressure (MCUP) and the etiological diagnosis of the urine loss. In the ultrasonography, the position of the bladder neck was evaluated in relation to the inferior border of the pubic symphysis, and its mobility as well as the diameter of the urethra and bladder neck. The women were categoriaed according to the urodynamic study in to stress urinary incontinence, detrusor instability and mixed urinary incontinence.

Results: 1) the bladder neck, at rest was most frequently above the inferior border of the pubic symphysis and, during effort, below or at the height of the bony reference, in the three groups; 2) the mobility of the bladder neck was similar in the groups; 3) there was no significant correlation between MCUP and the diameter of the urethra and of the bladder neck. Conclusion: we deem that ultrasonography of the bladder neck is always a complement to the clinical evaluation and the urodymanic study.

KEY WORDS: Urinary incontinence. Urodynamic study. Ultrasonography, transvaginal. Ultrasonography, urethra. 


\section{Referências}

1.Beco J, Sulu M, Scaaps JP, Lambotte R . Une nouvelle approche des troubles de continence chez la femme: l'échografie urodynamique par voie vaginale. J Gynecol Obstet Biol Reprod 1987; 16:987-98.

2.Hodgkinson CP. Relationships of female urethra and bladder in urinary stress incontinence. Am J Obstet Gynecol 1953; 65:560-73.

3.International Continence Society. Committee on Standardisation of Terminology - The standardisation of terminology of lower urinary tract function. In: Ostergard DR, Bent AE. Urogynecology and Urodynamics: Theory and Practice. 3rd. Baltimore: Williams \& Wilkins; 1991. p.545-62.

4.Jarvis GJ, Hall S, Stamp S, Millar DR, Johnson A. An assesment of urodynamic examination in incontinent women. Br J Obstet Gynaecol 1980; 87:893-6.
5.Kohorn EI, Scioscia AL, Jeanty P, Hobbins JC. Ultrasound cystourethrography by perineal scanning for the assessment of female stress urinary incontinence. Obstet Gynecol 1986; 68: 269-72.

6.Oliveira LM, Girão MJBC, Sartori MGF, Wakawaiachi VMB, Suguita MA, Gonçalves WJ, Baracat EC, Rodrigues DE LIMA G. Comparação entre o diagnóstico clínico e a avaliação urodinâmica em mulheres com incontinência urinária. Rev Bras Med Ginecol Obstet 1994, 5:310-2.

7.Ribeiro RM. Mobilidade de colo vesical em portadoras de incontinência urinária de esforço: estudo ultrasonográfico.Tese (Livre docência). São Paulo: Faculdade de Medicina da Universidade de São Paulo; 1992.

8.Souza AZ. Stress incontinence of urine. Int Surg 1976; 61:396-402.

9.White D, McQuown, D, McCarthy TA, Ostergard DR. Real Time Ultra-Sonography in the evaluation of urinary stress incontinence. Am J Obstet Gynecol $1980 ; 138: 235-7$.

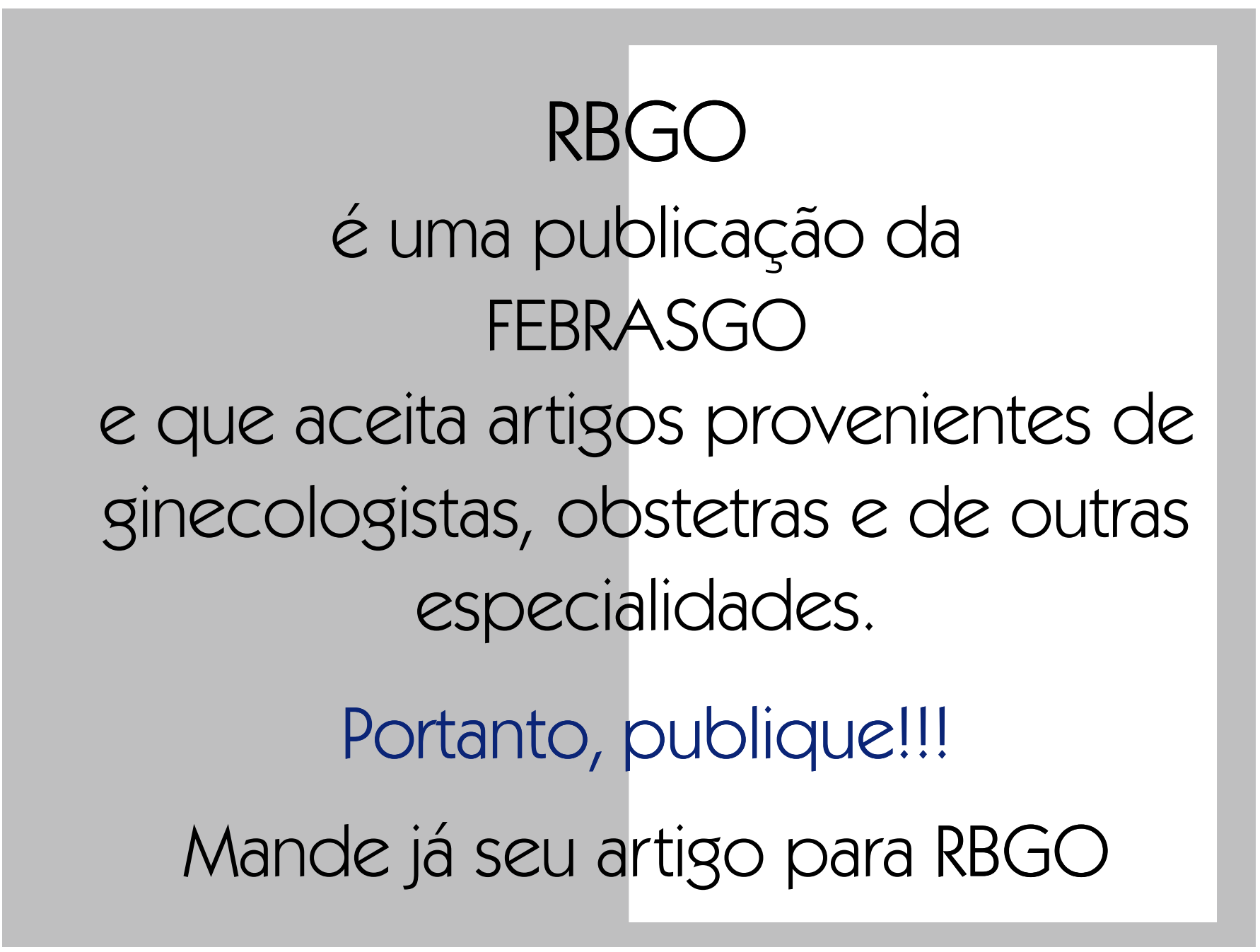

